\title{
Personal Resilience Can Be Well Estimated from Heart Rate Variability and Paralinguistic Features during Human-Robot Conversations
}

\author{
Shin-Min Hsu ${ }^{1,2}$, Sue-Huei Chen ${ }^{1}$ and Tsung-Ren Huang ${ }^{1,2,3, * D}$ \\ 1 Department of Psychology, National Taiwan University, Taipei 10617, Taiwan; \\ smhsu@mil.psy.ntu.edu.tw (S.-M.H.); shchen@ntu.edu.tw (S.-H.C.) \\ 2 MOST Joint Research Center for AI Technology and All Vista Healthcare, Taipei 10617, Taiwan \\ 3 MOST AI Biomedical Research Center, Tainan 70101, Taiwan \\ * Correspondence: tren@mil.psy.ntu.edu.tw
}

Citation: Hsu, S.-M.; Chen, S.-H.; Huang, T.-R. Personal Resilience Can Be Well Estimated from Heart Rate Variability and Paralinguistic Features during Human-Robot Conversations. Sensors 2021, 21, 5844. https://doi.org/10.3390/s21175844

Academic Editor: Ahmad Rad

Received: 5 July 2021

Accepted: 26 August 2021

Published: 30 August 2021

Publisher's Note: MDPI stays neutral with regard to jurisdictional claims in published maps and institutional affiliations.

Copyright: (c) 2021 by the authors. Licensee MDPI, Basel, Switzerland. This article is an open access article distributed under the terms and conditions of the Creative Commons Attribution (CC BY) license (https:// creativecommons.org/licenses/by/ $4.0 /)$.

\begin{abstract}
Mental health is as crucial as physical health, but it is underappreciated by mainstream biomedical research and the public. Compared to the use of AI or robots in physical healthcare, the use of AI or robots in mental healthcare is much more limited in number and scope. To date, psychological resilience - the ability to cope with a crisis and quickly return to the pre-crisis statehas been identified as an important predictor of psychological well-being but has not been commonly considered by AI systems (e.g., smart wearable devices) or social robots to personalize services such as emotion coaching. To address the dearth of investigations, the present study explores the possibility of estimating personal resilience using physiological and speech signals measured during humanrobot conversations. Specifically, the physiological and speech signals of 32 research participants were recorded while the participants answered a humanoid social robot's questions about their positive and negative memories about three periods of their lives. The results from machine learning models showed that heart rate variability and paralinguistic features were the overall best predictors of personal resilience. Such predictability of personal resilience can be leveraged by AI and social robots to improve user understanding and has great potential for various mental healthcare applications in the future.
\end{abstract}

Keywords: automatic personality recognition; human-robot interaction; personal resilience; physiological signals; speech signals

\section{Introduction}

\subsection{Background and Motivation}

People often take good care of their physical health while ignoring their mental health. Correspondingly, while many AI and robot applications for physical healthcare have been developed over the years, those used for mental healthcare applications are limited in number and scope. However, people neglect a crucial fact: like physical illness, mental illness can lead to death $[1,2]$.

Individuals with better protective factors are less likely to suffer from mental health problems, especially when facing traumatic or stressful life events. One of these protective factors is trait resilience [3], which is a positive personality characteristic indicative of one's adaptability in the face of adversity. For instance, trauma and adversity in childhood may negatively impact stress response systems [4] and contribute to mental disorders such as post-traumatic stress disorder (PTSD) and depression [5,6]. Nevertheless, resilient children can cope well with loss or trauma, not suffer from mental illnesses, and even thrive under such adverse life experiences $[7,8]$.

While an increasing number of psychological studies on resilience indicates a growing interest among researchers in this important topic, there are discrepancies as to how to 
define and conceptualize personal resilience. In the relevant literature, personal resilience can be discussed as a trait-like capacity, an adaptive process of overcoming adversity, or an outcome of (un)successful adaptation [9]. These different characterizations of personal resilience have led to incongruent resilience measures [10] and hence the difficulty of integrating divergent findings across studies [11]. Among the three characterizations of personal resilience, the trait-like capacity is the only one that precedes adversities from environments [12] and thus can be utilized for early identification and proactive protection of vulnerable populations. Here, the adversities can vary in severity and range from daily hassles to major life events [11], including ostensibly positive and yet stressful life events (e.g., job promotion or marriage) [9].

Despite the values of trait resilience, research on personality computing has mainly focused on estimating the Big Five personality traits [13,14]. For example, many studies have investigated the associations between the Big Five personality traits and physiological $[15,16]$ or linguistic features [17-20]. By contrast, only a handful of studies have sought biomarkers or behavioral markers of resilience. Moreover, these identified markers are either invasive measures (e.g., neurochemical or immune ones [21]) or linguistic patterns in written narratives (e.g., [22,23]), which are not easily accessible in daily scenarios. To add to the literature on personality computing, the present study set out to investigate whether the psychological resilience of a person can be accurately estimated using simple, cost-effective, and non-invasive procedures.

Specifically, we measured physiological and speech signals to be used as predictors in our resilience-estimating models. These physiological signals can be acquired by wearable devices (e.g., smartwatches or smart bands), and speech signals can be captured during human-human or human-robot conversations. In our resilience-predictive models, the physiology-based predictors included galvanic skin response (GSR), electrocardiograms (ECG), and heart rate variability (HRV); the speech-based predictors encompassed audio and linguistic features. While these physiological signals have been found to correlate with psychological resilience [21], speech markers of resilience, if any, are not yet identified. Therefore, the present study aimed to explore the use of speech signals for predicting personal resilience and compare the predictive powers of these two data sources.

\subsection{Theoretical Basis}

\subsubsection{Physiological Signals}

GSR, also known as skin conductance or electrodermal activity (EDA), is a biosignal indicating the change in skin conductance resulting from the autonomic activation of sweat glands. Controlled by the sympathetic nervous system, active sweat glands are driven by high-arousal events. When sweat glands are triggered and secrete fluid, the electrical current flow changes. This change leads to a variation in the skin conductance, as often measured by the electrical potential difference between a pair of electrodes placed in the hands or on the feet.

ECG signals are frequently utilized to reveal cardiac conditions. The heart rhythm is influenced by the autonomic nervous system (ANS), which consists of the sympathetic nervous system (SNS) and the parasympathetic nervous system (PNS). While the SNS influences the acceleration and excitation of the heart rate, the influence from the PNS is slow and inhibitory. The average heart rate results from a balance between the SNS and PNS, and the ratio of low- to high-frequency components of the ECG (i.e., LF/HF) indicates such a balance [24].

The ECG signal comprises a sequence of positive and negative waves. The first wave, the $\mathrm{P}$ wave, is generated by the depolarization of the atria. The following QRS complex represents the depolarization of both ventricles. The last $\mathrm{T}$ wave represents ventricle repolarization. As illustrated in Figure 1, the amplitudes of these ECG waves were used in our analyses. 


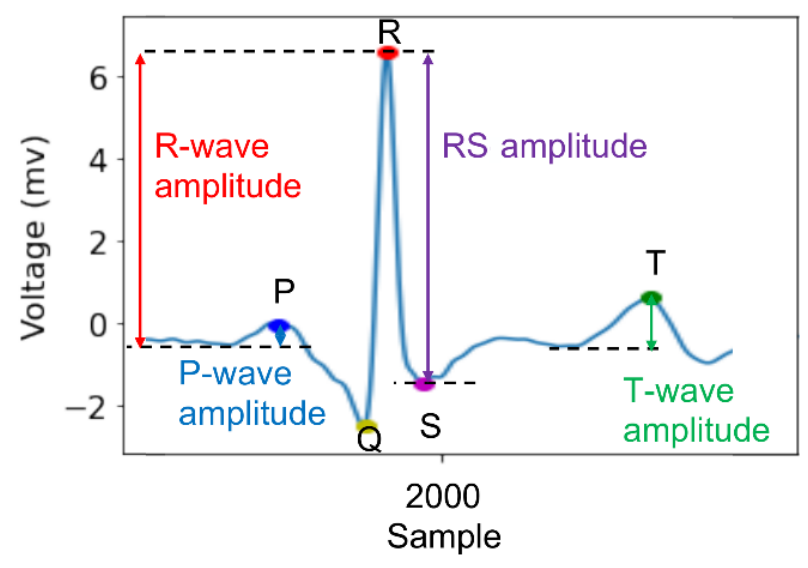

Figure 1. Illustration of ECG waves.

Besides the ECG amplitudes, heart rate variability (HRV) is often computed from ECG signals because it is a strong indicator of one's cardiac or general health condition. Different indices can be derived from HRV. Time-domain features are extracted based on the variation in the time interval between consecutive heartbeats, such as normal-to-normal $(\mathrm{NN})$ intervals and intervals between adjacent QRS complexes. Frequency-domain features, such as spectrograms, are also frequently examined.

Although few studies have discussed the direct relationship between resilience and physiological signals in healthy individuals, such a relationship may be inferred from the known associations between PTSD and ECG. When faced with traumatic events, less resilient individuals are more likely to suffer from PTSD [25], the ECG characteristics of which include a higher heart rate, lower HF, and higher LF/HF [26,27]. Note, however, that although PTSD is often conceptualized as a category of mental disorder, it can be viewed as a spectrum disorder in terms of symptom severity, the nature of the stressor, and responses to trauma [28]. Under such a dimensional conceptualization of PTSD, less resilient individuals that are not diagnosed with full PTSD may, to some extent, also show the aforementioned ECG characteristics, which suggest an elevated sympathetic activity and/or an attenuated parasympathetic activity.

\subsubsection{Speech Signals}

Speech conveys information through verbal and nonverbal channels. A speaker can communicate meaning not only by verbal contents per se but also by vocal techniques, such as modification of volume, prosody, and intonation (for a review, see [29]). More importantly, while a speaker expresses meaning consciously through these two communication channels, paralinguistic and linguistic features unconsciously expressed during a conversation may reveal the psychological traits or states of the speaker, such as personality or emotional states [30]. Therefore, the current study leveraged paralinguistic and linguistic features extracted from speech signals to estimate psychological resilience.

Paralinguistic features can be further divided into physical and perceptual features [31]. The former is calculated directly from sound waves, including energy function/spectrum and cepstral coefficients; the latter is related to the human perception of the sound, such as loudness, pitch, and rhythm.

As for linguistic features, Linguistic Inquiry and Word Count (LIWC) [32] is a computerized language analysis program widely used to count linguistically or psychologically meaningful words in texts. These words are either content words (e.g., words related to positive emotion or social activities) or function words (e.g., articles, pronouns, and prepositions). While content words deliver semantic meaning, function words partly form linguistic styles [33]. As an example of differences in linguistic style, written texts, relative to spoken texts, are shorter in general but use longer words, more attributive adjectives, and a more varied vocabulary [34]. Therefore, it is unclear how well the linguistic features 
of resilience found in written texts (e.g., [22,23]) can be used by social robots to estimate personal resilience from speech.

\section{Materials and Methods}

This section presents the materials and methods employed in the study. Section 2.1 describes the study procedure, including the resilience questionnaire, signal recording method, social robot, and task instructions. Section 2.2 introduces the features extracted from physiological and speech signals. Section 2.3 details the feature selection method. Section 2.4 then presents the results from various resilience-estimating models.

\subsection{Procedure}

The whole study took approximately $1.5 \mathrm{~h}$ to complete, and each participant was paid \$240 New Taiwan Dollars as compensation for research participation. After giving written informed consent of being video- and sensor-recorded, each participant completed a questionnaire that measures personal resilience. Then, the participant was invited by a social robot to recall autobiographical memory with her/his video and physiological signals simultaneously recorded.

\subsubsection{Participants}

Thirty-five students from National Taiwan University volunteered to participate in the study, but three were excluded from further analysis because they did not follow the instructions. The final sample size was 32 participants (18 females, age range 20-29, $\mathrm{M}=22.91, \mathrm{SD}=2.41$ ).

\subsubsection{Questionnaires}

While there is no " gold standard" among different measures of resilience, three resilience scales-the Brief Resilience Scale (BRS) [35], the Connor-Davidson Resilience Scale (CD-RISC) [36], and the Resilience Scale for Adults (RSA) [37] — have been found to be psychometrically better than the others [38]. In the present study, we favored the RSA over the other two because the 5-dimensional RSA is a more elaborated measure of resilience than the 1-dimensional BRS and was developed more generally for the healthy population than the more clinically oriented CD-RSIC.

The current study employed the Mandarin Chinese version of the 33-item RSA, which was constructed using a back-translation procedure by the author of this article (S.-H.C.). An exploratory factor analysis of the Mandarin Chinese version partially recovered the five-factor structure of RSA from 29 items: personal strength (6 items), family cohesion (7 items), social resources ( 8 items), social competence (4 items), and structured style (4 items). The Cronbach's alphas of the five factors were $0.92,0.83,0.87,0.85$, and 0.85 , respectively. The overall test-retest correlation of the Mandarin Chinese RSA was 0.89 for an interval of 3-4 weeks.

\subsubsection{Signal Recording}

We recorded both physiological signals and speech during each human-robot interaction session. Two types of physiological signals-electrocardiography (ECG) and galvanic skin response (GSR)-were measured with a NeXus-10 system (Mind Media $\mathrm{BV}$, Roermond-Herten, Netherlands), which was controlled by the BioTrace+ Software. The ECG signal was measured with an ExG sensor. Following the ECG wrist placement tutorial of the BioTrace+ Software, the ECG electrodes were placed on every participant's arms. One was placed on the left arm as a reference, one was placed on the lower part of the left arm as the positive channel, and one was attached to the lower part of the right arm as the negative channel. For GSR recording, two electrodes of a GSR sensor were attached separately to the index and ring finger on each participant's right hand. These signals were monitored on NeXus-10 at a rate of 2048 samples per second. The speech was recorded using Digital Video Recorder H.264 DVR. 


\subsubsection{Human-Robot Interaction}

We used a humanoid social robot-RoBoHoN (Sharp Co., Ltd., Sakai, Japan)—as the conversational agent in the present study. RoBoHoN is a programmable robot with built-in speech-to-text and text-to-speech engines. Nevertheless, at times it misrecognizes spoken words in Mandarin Chinese and speech pauses/endpoints, thereby making inappropriate responses. Because such machine errors might induce negative emotions in the participants and hence become confounding factors, we followed the convention of human-robot studies to adopt a Wizard-of-Oz approach for precisely controlling, via wireless internet, when and what the social robot spoke. Specifically, the social robot functioned as a client who received Remote Procedure Calls (RPCs) of text-based speech commands from a remote computer server. The server offered a web interface for human operators to quickly select predefined sentences (e.g., memory retrieval instructions detailed in the next section) or manually enter on-the-fly texts to be spoken by the social robot. While a participant interacted with the social robot in a well-lit experimental room, the experimenters stayed in a separate room, remotely monitoring the participant's responses via a real-time video camera and operating the social robot via the web-based control panel. According to our post-study inquiry, all the participants thought the social robot to be under active development and completely autonomous.

\subsubsection{Memory Retrieval}

To set up the experiment like a casual conversation during a human-robot interaction in healthcare settings, our social robot expressed interest in learning a participant's personal history and asked each participant to recall memories from his or her life. Six types of memories about social interaction experiences were queried: a positive or a negative memory from either childhood (7-12 years old), adolescence (13-18 years old), or early adulthood (19-24 years old) period. The order of these six queries was randomized for each participant.

The social robot delivered all the memory retrieval instructions, which were adapted from an emotional memory study [39]: "Please describe a memory that related to a positive [negative] experience in a social interaction context during your childhood [adolescence, early adulthood]. The memory should be associated with strong feelings and be something you have thought about many times." Moreover, each participant was required to recall in steps to separate different cognitive processes, which may be associated with different physiological and/or linguistic features. These steps were (1) memory selection (selecting a specific memory for one minute), (2) memory immersion (immersing oneself in the memory for two minutes), and (3) memory description (describing the memory to the robot without time limit). For the purpose of the current study, only data from the third step were analyzed.

\subsection{Feature Extraction}

\subsubsection{Physiological Signals}

We applied the first version of the Python package NeuroKit [40] to process ECG signals, including HRV calculation and wave locating. Specifically, ECG signals were first filtered with a Butterworth high-pass filter and transformed into z scores. Then, six HRV features were extracted and directly adopted.

We calculated five ECG amplitudes on our own to be used as resilience-predicting features because NeuroKit could locate R-, T-, P-, Q-, and S-wave peaks but did not provide the corresponding amplitude values relative to baselines (Figure 2a). Specifically, the baseline voltage preceding a P-or T-wave was averaged from samples 97.65 to $195.31 \mathrm{~ms}$ preceding each $\mathrm{P}$ or $\mathrm{T}$ peak and then subtracted from the corresponding peak voltage to obtain the amplitude of each P- or T-wave. Similarly, the baseline voltage preceding an Rwave was averaged from samples 350 to $300 \mathrm{~ms}$ preceding each $\mathrm{R}$ peak and then subtracted from the corresponding peak voltage to obtain the amplitude of each R-wave. The QR 
amplitude is the absolute difference between the Q- and R-peaks; the RS amplitude is the absolute difference between the R- and S-peaks.

Moreover, we used the Python package PySiology [41] to automatically extract five GSR features, as shown in Figure 2b. The rise time is the time during which a GSR rises, from start to apex; the decay time is the time during which a GSR decays to $50 \%$ of the amplitude. The GSR amplitude is the value difference between the apex and the start of a GSR. The GSR width is the time interval from 50\% amplitude on the increasing side to $50 \%$ amplitude on the declining side.

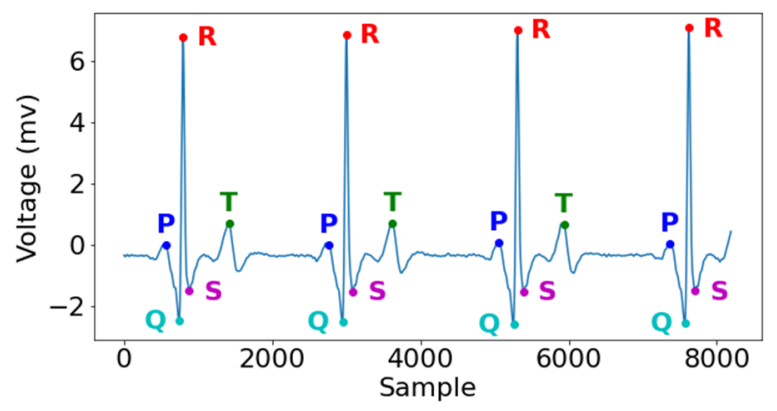

(a)

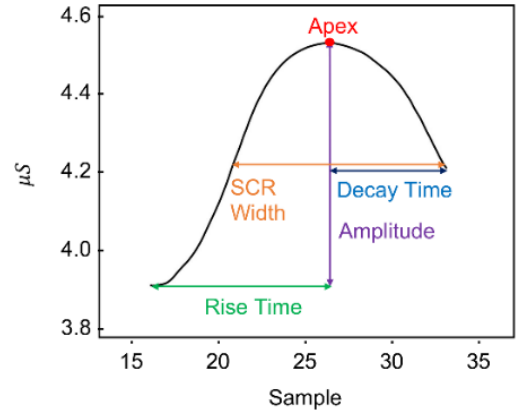

(b)

Figure 2. (a) Peaks in ECG signals located by the NeuroKit. (b) Four GSR features extracted by PySiology.

The aforementioned features, together with their standard deviations, then became the final outputs of our feature extraction process. In total, there were five HRV features, ten ECG features, and ten GSR features for later model training and testing, as summarized in Table 1.

Table 1. HRV, ECG, and GSR features.

\begin{tabular}{cccc}
\hline & HRV Features & ECG Features & GSR Features \\
\hline Features & Meaning & Features & Features \\
\hline meanNN & The mean of normal-to-normal (NN) intervals & P mean \& std & Rise Time mean \& std \\
SDNN & The standard deviation of the NN intervals & R mean \& std & Amplitude mean \& std \\
RMSSD & The root mean square of the RR intervals & T mean \& std & Apean \& std \\
LF & Power in the low-frequency range $(0.04-0.15 \mathrm{~Hz})$ & QR mean \& std & Decay Time mean \& std \\
HF & Power in the high-frequency range $(0.15-0.40 \mathrm{~Hz})$ & WS mean \& std & Width mean \& std \\
LF/HF & The ratio of LF to HF & & \\
\hline
\end{tabular}

\subsubsection{Speech Signals}

To analyze paralinguistic features in speech, we extracted the Computational Paralinguistics Challenge 2013 (ComParE2013) feature set [42] using the OpenSMILE toolkit [43]. This set contains 6373 acoustic features comprising 65 low-level descriptors (LLDs) and their functionals, as well as five temporal statistics. The LLDs include energy (intensity), spectral, cepstral (MFCC), voice quality (e.g., jitter), shimmer, harmonics-to-noise ratio (HNR), spectral harmonicity, and psychoacoustic spectral sharpness. We have also tested our prediction models with the eGeMAPS feature set [44], which comprises only 88 acoustic features but is not as predictive of resilience as the ComParE2013 features.

To analyze linguistic features in speech, we manually prepared verbatim transcripts of the memories described by the participants and applied CkipTagger [45] to word-segment these Mandarin Chinese transcripts. Then, we adopted the Linguistic Inquiry and Word Count Dictionary for Traditional Chinese (TC-LIWC 2007) to calculate the proportion or relative frequency of 72 psychologically or linguistically meaningful word categories in these transcripts. 


\subsection{Feature Selection}

Although mutually correlating features pose the problem of multicollinearity, these mutual correlations do not, in general, affect prediction performances [46]. For example, for an underlying data relationship $Y=X_{1}+X_{2}$ where $X_{1}=X_{2}$ (i.e., a perfect correlation between $X_{1}$ and $X_{2}$ ), the estimates of $\beta_{1}$ and $\beta_{2}$ in the regression model $Y=\beta_{1} X_{1}+\beta_{2} X_{2}$ are unreliable for interpreting the relative importance of $X_{1}$ and $X_{2}$ in predicting Y. However, an infinite number of such regression models can make equally perfect predictions as long as $\beta_{1}+\beta_{2}=2$. In the current study, when building resilience-predicting models, we were more concerned with the predictive power than the relative importance of features within each modality. Therefore, before model-building, we did not use any dimensionality reduction methods or exclude any features to address the multicollinearity problem.

When constructing multivariate predictive models, we used Pearson's correlation as a relevance index for univariate feature selection. Specifically, we computed the Pearson's correlation coefficients between the RSA resilience scores and the features of each modality (i.e., GSR, HRV, ECG, paralinguistic, and linguistic features) and sorted the features according to the absolute values of these coefficients. To achieve the best possible prediction outcome, we treated the number of features for each modality as a model hyperparameter, which was then tuned during the model optimization stage, as detailed in the following section. Finally, each feature was standardized before model training or testing.

\subsection{Model Training and Testing}

For each single-modal resilience-predicting model, the number of selected features was explored with a cap of 6,10,10,100, and 72 features for the HRV, ECG, GSR, paralinguistic, and linguistic modality, respectively. For example, because there were six HRV features before feature selection, six possible sets of HRV features could be constructed after the feature sorting - top six features, top five features, top four features, etc.- -and only results from the best-performing HRV feature set were reported.

Two multi-modal feature sets were defined based on their availability in real-life scenarios. The physiological superset combined the best-performing GSR, HRV, and ECG feature sets, as they can be captured by wearable devices; the speech superset combined the best-performing paralinguistic and linguistic feature sets, as they can be obtained from verbal conversations.

For each research participant and each designated feature set, one feature vector could be extracted to characterize each of the participant's six memory recall episodes ( 3 life periods $\times 2$ emotional valences). Therefore, there were six feature vectors associated with each participant. To find common patterns in these feature vectors from different memory episodes, all the resilience-predicting models in the current study treated these feature vectors equally as different data samples of the same participant. Moreover, these feature vectors of the same person were grouped to appear only during training or testing but not both for person-level cross-validation.

As for the level of personal resilience, we adopted a classification rather than regression approach when building our resilience-predicting models because of our relatively small number of participants $(\mathrm{N}=32)$. To generate the classification labels, we dichotomized each of the five RSA subscale scores into high vs. low groups based on the median, and the median scores were removed from further analysis.

Four types of machine-learning classifiers were then optimized for comparison: K-Nearest Neighbor (KNN), Logistic Regression (LR), Support Vector Machine (SVM), and Random Forest (RF). These models were trained and tested using 10-fold crossvalidation. The hyperparameters of each model were tuned via a grid search to obtain the best average F1-score. For the linear SVM, the best parameter $C$ was determined over $[1,11]$. The best number of neighbors for KNN was chosen over [1,10]. The best parameter $C$ for logistic regression was chosen among $0.001,0.01,0.1,1,10$, and 100 . For the random forest, the number of trees was chosen among $10,15,20,25$, and 30 . The minimum number of 
samples required to split an internal node was determined over [3,7], and the minimum number of samples required to be at a leaf node was explored from 1 to 3 .

\section{Results}

Here we present the descriptive statistics of the RSA scores in Section 3.1, the correlations between the RSA scores and personality dimensions as well as physiological/speech features in Section 3.2, and the classification accuracies of the resilience-predicting models in Section 3.3.

Paired sets of results will be presented. The first set corresponds to analyses of data collected during the three recalls of negative memories, whereas the second set corresponds to analyses of all the six recalls of positive and negative memories. The second sets of results are of both theoretical and applied interest. Theoretically, they are verifications of the hypothesis that ostensibly positive events can also be relevant in defining resilience [9]. For applications, it will be convenient to estimate one's resilience level regardless of narrative contexts.

\subsection{Personal Resilience}

As mentioned in Section 2.4, for each resilience dimension, the 32 participants were divided into high- and low-score groups. The total, median, mean, and standard deviation of the RSA scores are summarized in Table 2 for both the high- and low-score groups.

Table 2. Summary statistics of the RSA scores $(\mathrm{N}=32)$.

\begin{tabular}{cccccc}
\hline Resilience & PS $(\mathbf{M} \pm \mathbf{S D})$ & FC $\mathbf{( M} \pm \mathbf{S D})$ & SR $(\mathbf{M} \pm \mathbf{S D})$ & SC $(\mathbf{M} \pm \mathbf{S D})$ & $\mathbf{S S}(\mathbf{M} \pm \mathbf{S D})$ \\
\hline Median/Total & $30 / 42$ & $35.5 / 49$ & $44 / 56$ & $17.5 / 28$ & $20 / 28$ \\
High Group & $34.00 \pm 3.20$ & $39.56 \pm 3.12$ & $48.44 \pm 3.33$ & $22.88 \pm 2.25$ & $23.25 \pm 2.86$ \\
Low Group & $21.40 \pm 5.26$ & $28.44 \pm 4.82$ & $36.93 \pm 3.91$ & $14.06 \pm 2.29$ & $14.58 \pm 2.50$ \\
\hline
\end{tabular}

Note. $\mathrm{PS}=$ Personal Strength, FC = Family Cohesion, $\mathrm{SR}=$ Social Resources, $\mathrm{SC}=$ Social Competence, $\mathrm{SS}=$ Structured Style.

\subsection{Correlational Analysis}

The Pearson correlation coefficients between each RSA score and each resiliencepredicting feature are summarized in Figures 3-6. These correlation matrices were sorted based on the results of hierarchical clustering so that more correlated variables were arranged closer to each other in the matrices. Such matrix sorting helps to reveal the structures of correlations between RSA scores and their potential predictors in this explorative study.

\subsubsection{Big-Five Personality}

The correlations between RSA and personality scores are summarized in Figure 3. These results are consistent with the previous finding that RSA directly measures a welladjusted personality profile [47]. For example, social competence was positively correlated with extraversion and openness, structured style was positively correlated with conscientiousness, and personal strength was negatively correlated with neuroticism. In other words, a person high in RSA scores is likely to be an emotionally stable extravert who is also conscientious and open to new experiences.

Note also from Figure 3 that the five RSA dimensions were not independent of each other. Specifically, dimensions related to personal characteristics-social competence, structured style, and personal strength-were mutually associated as a group, whereas dimensions related to environmental factors-family cohesion and social resources-were covaried as another group. This correlation structure reflects the fact that RSA was designed to measure protective factors of resilience along both intrapersonal and interpersonal dimensions [37]. 


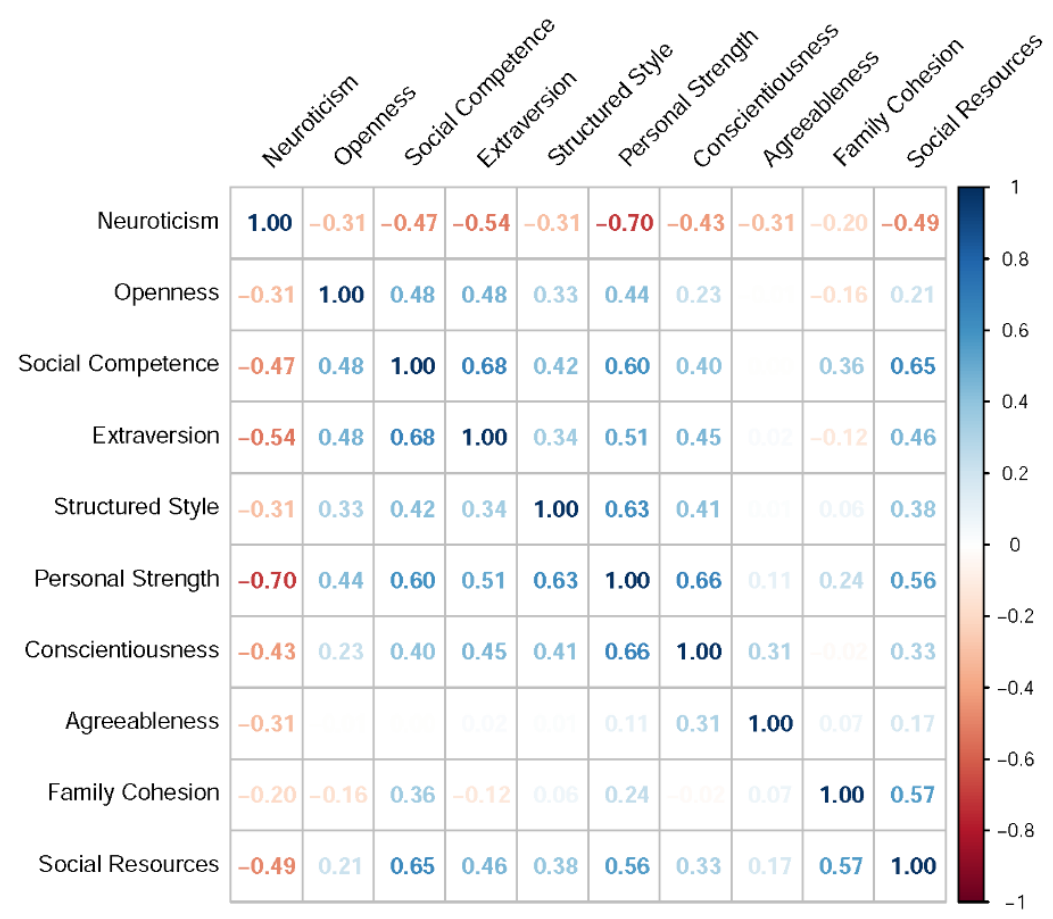

Figure 3. The correlations between each RSA score and each personality dimension.

\subsubsection{Physiological Features}

As shown in Figure 4a,b, among all the physiological features, two HRV featuresthe low-frequency component (i.e., LF) and the standard deviation of the NN intervals (i.e., SDNN)-are most associated with all the five resilience dimensions, especially social resources. Here, the higher LF and SDNN in less resilient individuals might indicate a higher sympathetic and / or low parasympathetic nervous activity, as suggested by previous studies $[26,27,48]$ under the dimensional conceptualization of PTSD.

Among all the ECG features, the mean amplitude of the T-wave (i.e., T mean) appears to be most indicative of resilience, particularly social resources. The negative correlation between $\mathrm{T}$ mean and resilience is in line with the finding that the T-wave amplitude is positively associated with psychological stress [49], which is, however, less experienced by more resilient individuals [9].

Among all the GSR features, the variability of rise time, apex, and amplitude (i.e., RT std, AP std, and AM std) are most indictive of resilience, particularly personal strength and structured style. These findings are consistent with a body of literature showing GSR to reflect one's emotionality. For example, emotionally evocative pictures can induce larger and more prolonged GSRs in more neurotic individuals [50]. It is likely that less resilient individuals may also show such GSR characteristics and their associated signatures in variability. Specifically, we found resilience to positively correlate with RT std but negatively correlate with the AP/AM std. 


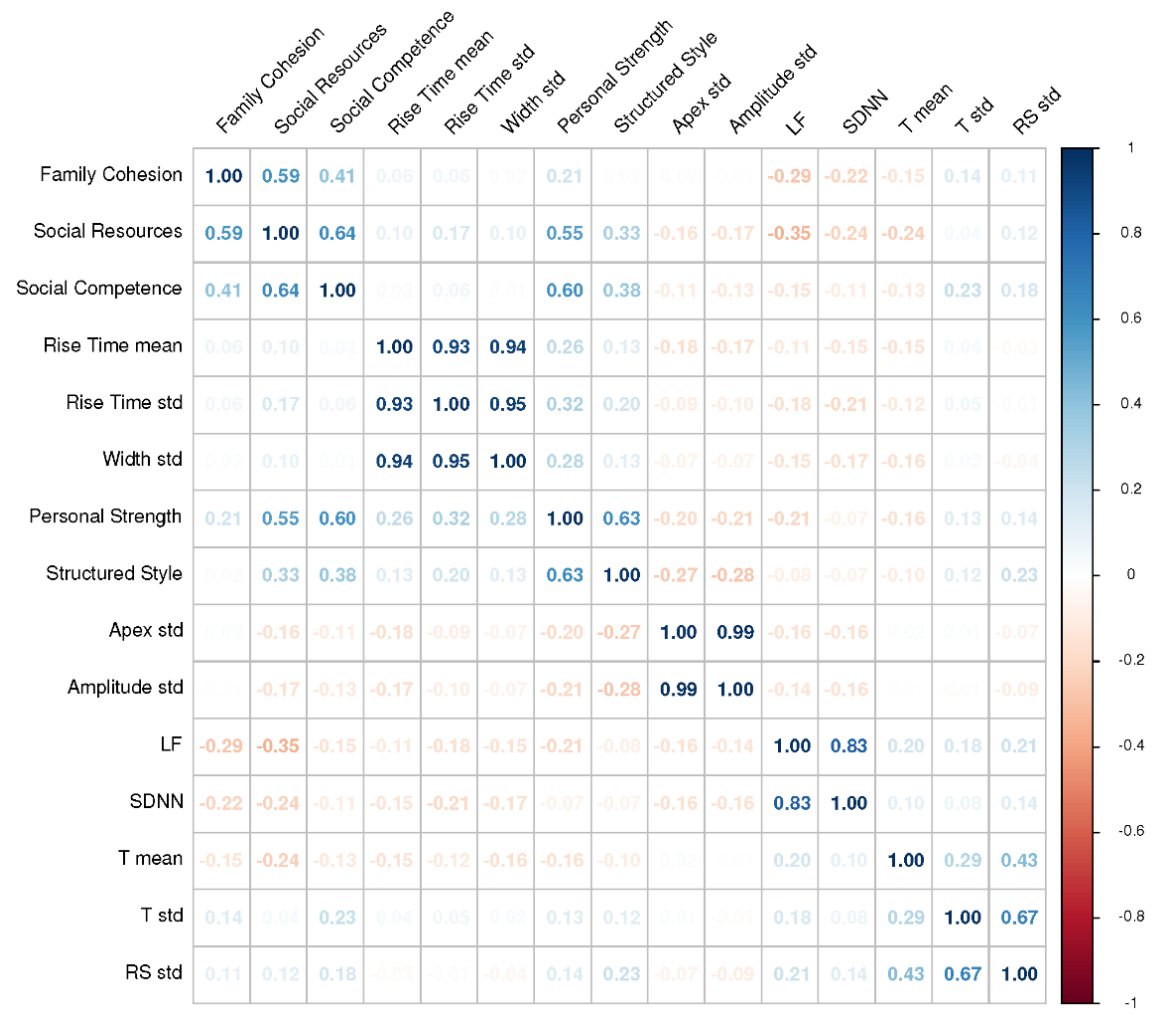

(a)

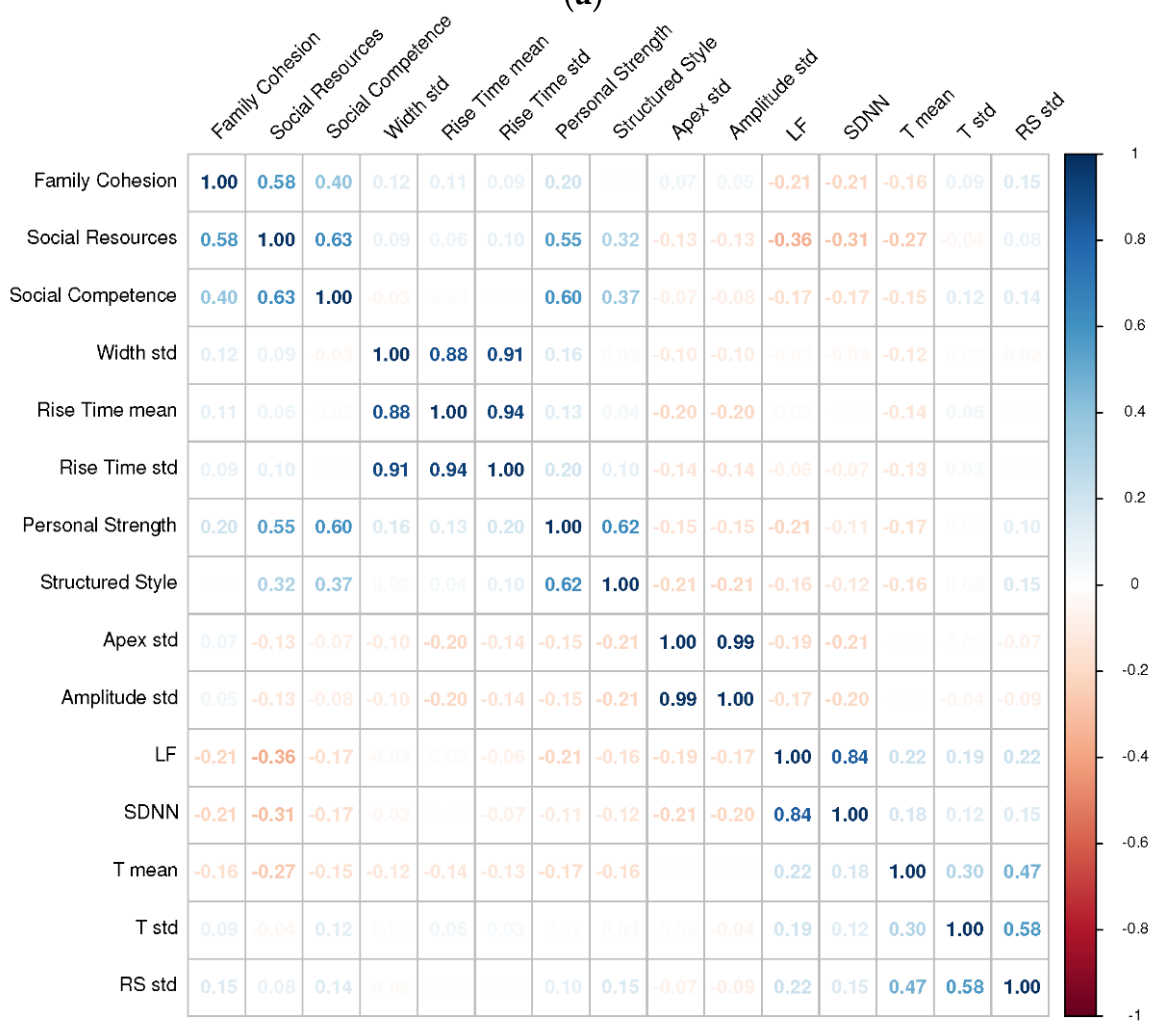

(b)

Figure 4. (a). The correlations between each RSA score and each physiological feature (only negative memories). Physiological features that were not significantly correlated with any RSA dimensions (i.e., $p>0.05$ ) are excluded from the plot. (b). The correlations between each RSA score and each physiological feature (positive and negative memories). Physiological features that were not significantly correlated with any RSA dimensions (i.e., $p>0.05$ ) are excluded from the plot. 


\subsubsection{Paralinguistic Features}

Among all the 6373 acoustic features, only the ones that were most correlated with RSA scores are reported in Figure 5a,b. Overall, the most resilience-relevant features are spectral features, including ones related to the fundamental frequency $\left(\mathrm{F}^{*}\right)$, auditory spectrum coefficients (audspec $\left.{ }^{*}\right)$, Mel Frequency Cepstral Coefficients $\left(\mathrm{mfcc}^{*}\right)$, spectral slope (pcm_fftMag_spectralSlope*), spectral flux (pcm_fftMag_spectralFlux $\left.{ }^{*}\right)$, and spectral harmonicity (pcm_fftMag_spectralHarmonicity*). The correlations between these spectral features with RSA scores may have to do with individuals' emotional styles unconsciously expressed through speech. In the literature of affective computing, it has been found that F0 and spectral distribution are important cues of affective states [51], and MFCC and spectral flux are particularly informative of emotional valence [44]. Given that emotions are regulated by resilience [9,52], resilience dimensions can then be linked with these spectral features.

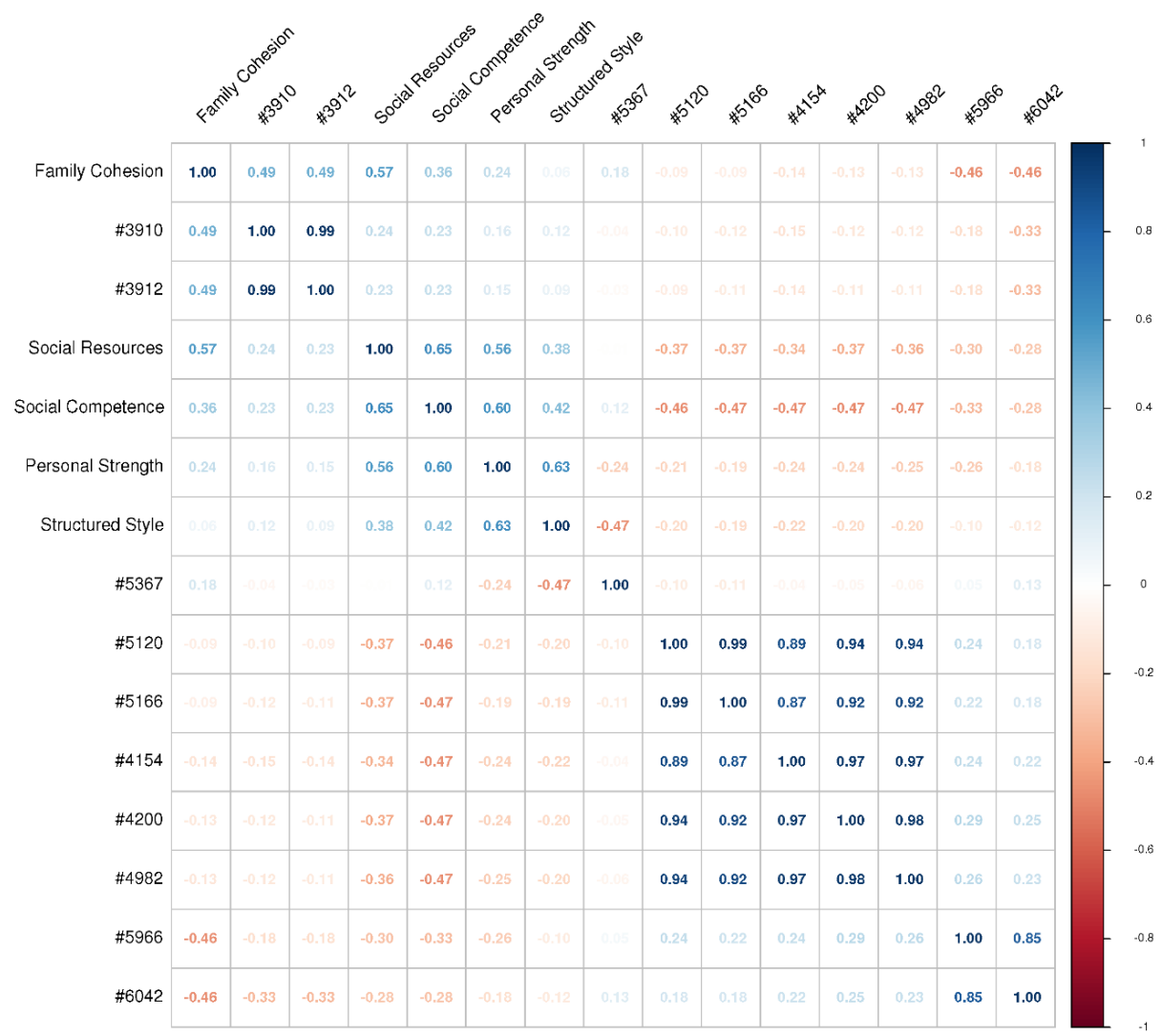

(a)

Figure 5. Cont. 


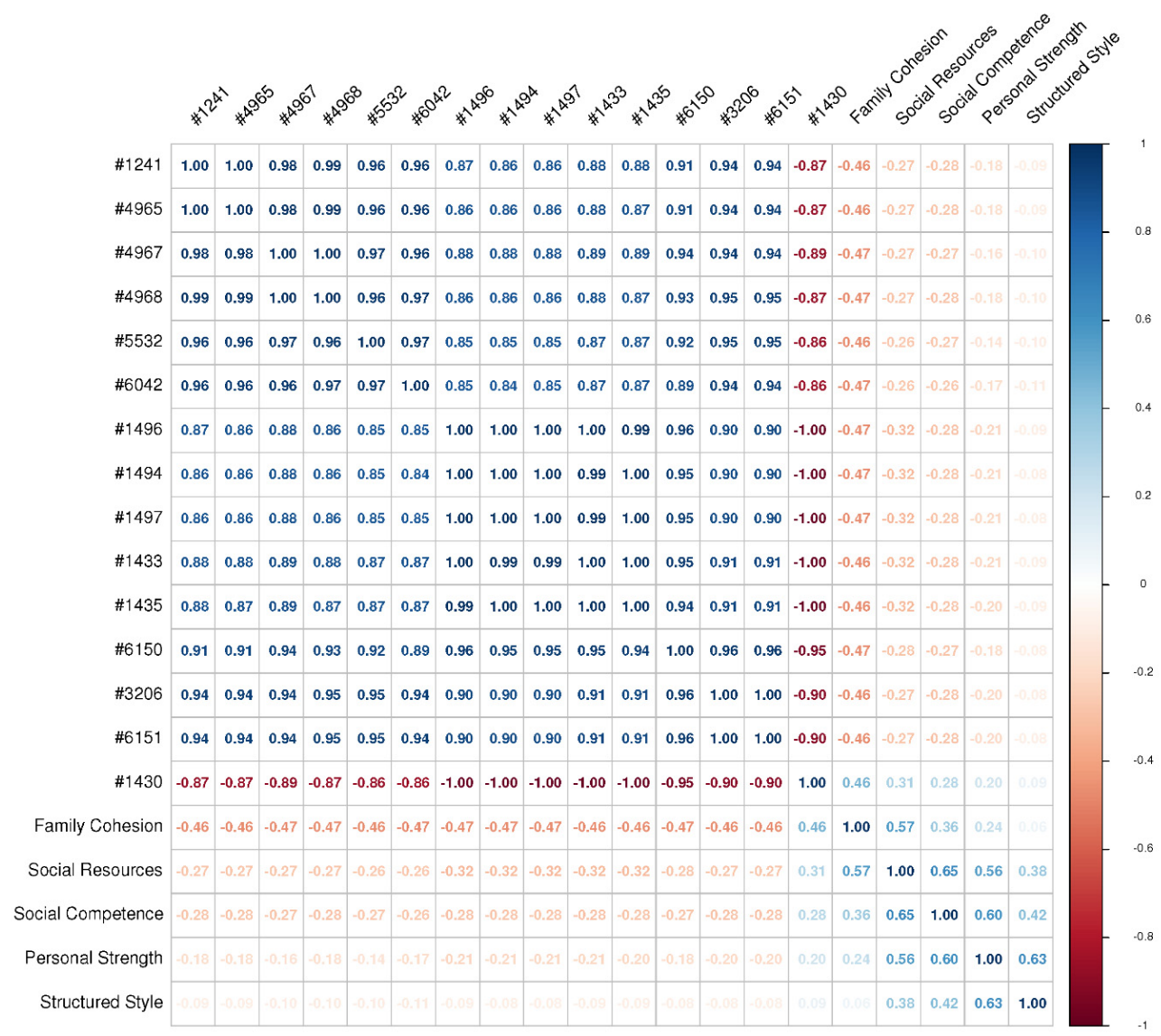

(b)

Figure 5. (a). The correlations between each RSA score and each ComParE2013 paralinguistic feature (only negative memories). Because there were many statistically significant correlations $(p<0.05)$, only paralinguistic features with at least one $|\mathrm{r}|>0.46$ were included in this plot. \#3910 = F0final_sma_de_quartile3; \#3912 = F0final_sma_de_iqr2-3; \#5367 = mfcc_sma[9]_linregc2; $\# 5120=$ pcm_fftMag_spectralSlope_sma_centroid; \#5166 = pcm_fftMag_spectralHarmonicity_sma_ centroid; \#4154 = audspec_lengthL1norm_sma_centroid; \#4200 = pcm_RMSenergy_sma_centroid; $\# 4982$ = pcm_fftMag_spectralFlux_sma_centroid; \#5966 = pcm_fftMag_fband1000-4000_sma_de_ stddevRisingSlope; \#6042 = pcm_fftMag_spectralFlux_sma_de_meanFallingSlope. (b). The correlations between each RSA score and each ComParE2013 paralinguistic feature (positive \& negative memories). Because there were many statistically significant correlations $(p<0.05)$, only paralinguistic features with at least one $|\mathrm{r}|>0.46$ were included in this plot. \#1241 = pcm_fftMag_spectralFlux_sma_range; $\# 4965$ = pcm_fftMag_spectralFlux_sma_peakRangeAbs; \#4967 = pcm_fftMag_spectralFlux_sma_ peakMeanAbs; \#4968 = pcm_fftMag_spectralFlux_sma_peakMeanMeanDis; \#5532 = pcm_RMSenergy _sma_de_meanFallingSlope; $\# 6042=$ pcm_fftMag_spectralFlux_sma_de_meanFallingSlope; $\# 1496=$ pcm_ fftMag_spectralHarmonicity_sma_iqr2-3; \#1494 = pcm_fftMag_spectralHarmonicity_sma_quartile3; $\# 1497=$ pcm_fftMag_spectralHarmonicity_sma_iqr1 -3 ; \#1433 = pcm_fftMag_spectralSlope_sma_ iqr1-2; \#1435 = pcm_fftMag_spectralSlope_sma_iqr1-3; \#6150 = pcm_fftMag_spectralHarmonicity_ sma_de_posamean; \#3206 = pcm_fftMag_spectralHarmonicity_sma_de_stddev; \#6151 = pcm_fftMag_ spectralHarmonicity_sma_de_rqmean; \#1430 = pcm_fftMag_spectralSlope_sma_quartile1.

\subsubsection{Linguistic Features}

Among all the 72 TC-LIWC categories, only word categories most correlated with RSA scores are reported in Figure $6 \mathrm{a}, \mathrm{b}$. The detailed descriptions and exemplars of each category can be found elsewhere [53]. Note first from the figures that hierarchically organized TC-LIWC categories are not independent of each other, and the correlations among TC-LIWC categories partially reflect their overlapping vocabularies (e.g., sad and 
negative emotion, we and social, auxiliary verbs and discrepancy words, etc.) and partially reflect the structures among their corresponding psychological dimensions. Secondly, while only a few physiological features were correlated with resilience, several linguistic features covaried with different dimensions of resilience. These results echo the findings that LIWC is generally sensitive to detect individual differences in terms of attentional focus, thinking styles, emotionality, and social relationships [54].

While some of the RSA-LIWC correlations are sensible (e.g., Family Cohesion and family words, Social Competence/Resources and social words, etc.), negative emotion words (e.g., sad) are unexpectedly used more by highly resilient individuals in the present study. Although past studies have often shown that resilient people thrive through positive emotions [52,55], resilient people do respond to negative events initially with negative affect [52]. Empirical evidence further suggests that trait resilience is not well characterized by unconditional positive emotions but by the ability to flexibly switch emotional responses to match the environmental demands [56]. Other studies that analyzed written texts (e.g., diaries in [22]) might observe more frequent use of positive emotions by highly resilient individuals as a result of their positive appraisal of events [9]. By contrast, our memory immersion procedure might vividly reactivate negative memories of the participants and thereby accentuate the characteristics of their emotional reactivity rather than cognitive appraisal. Future studies can further investigate such a possibility of procedural/contextual influences on linguistic features in association with resilience.

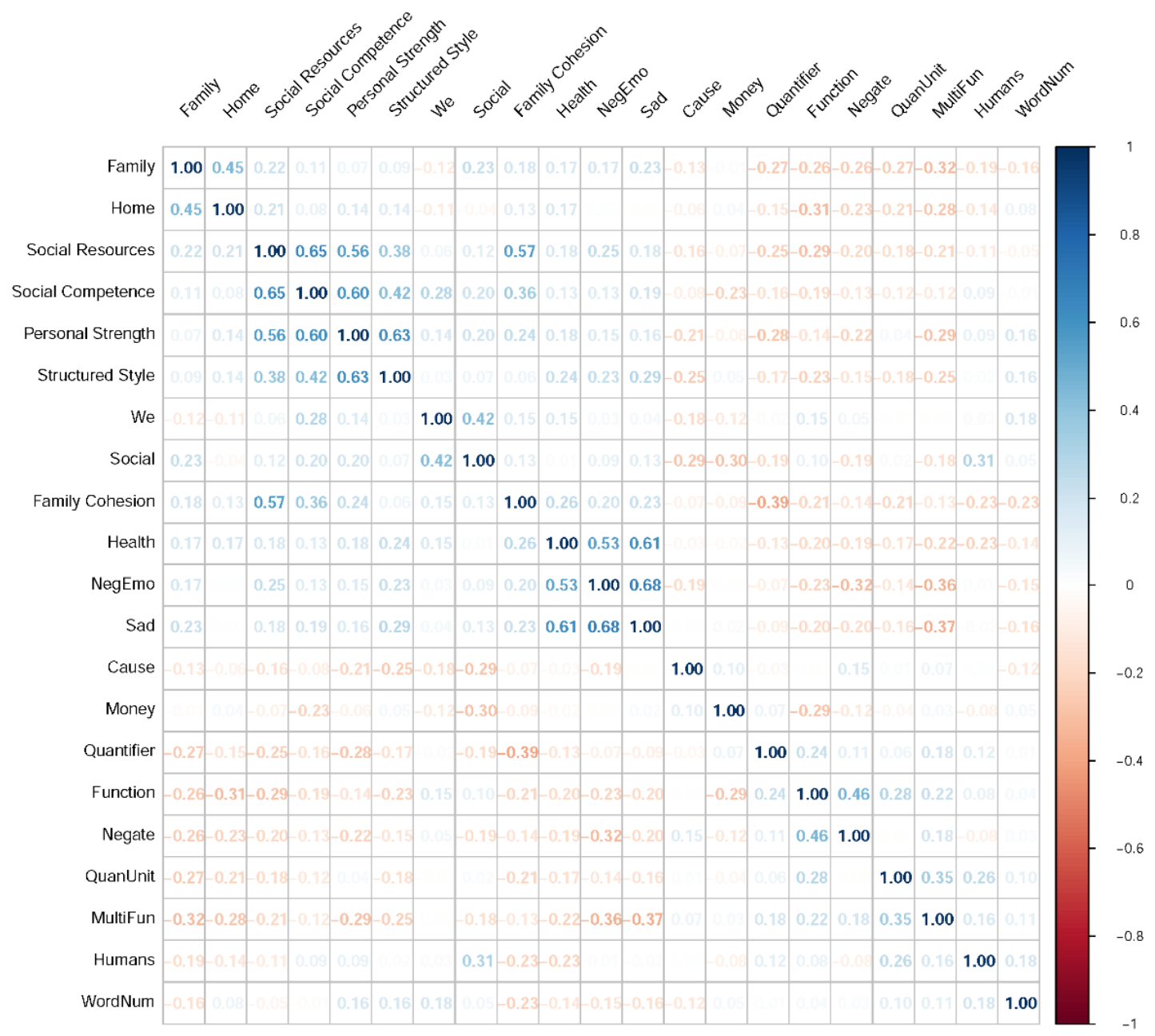

(a)

Figure 6. Cont. 


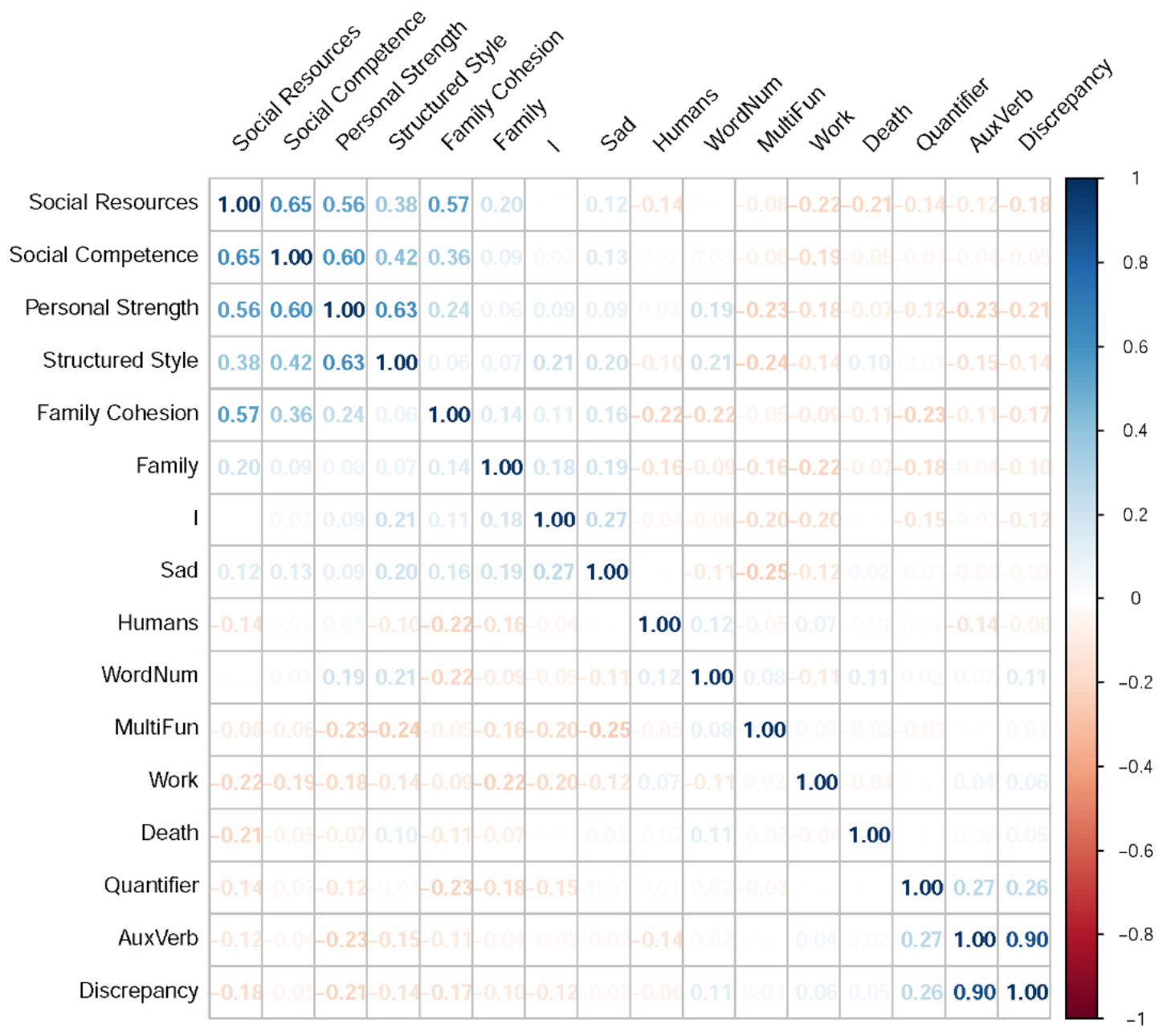

(b)

Figure 6. (a). The correlations between each RSA score and each TC-LIWC linguistic feature (only negative memories). Because there were many statistically significant correlations $(p<0.05)$, only linguistic features with at least one $|\mathrm{r}|>0.2$ were included in this plot. MultiFun = multifunction words; NegEmo = negative emotion; QuantUnit = quantity unit; WordNum = number of words (b). The correlations between each RSA score and each TC-LIWC linguistic feature (positive and negative memories). Because there were many statistically significant correlations $(p<0.05)$, only linguistic features with at least one $|\mathrm{r}|>0.2$ were included in this plot. AuxVerb = auxiliary verbs; MultiFun = multifunction words; NegEmo = negative emotion; WordNum = number of words.

\subsection{Classification Results}

Tables 3 and 4 present the mean F1-scores of resilience predictions using different modalities with different types of classifiers. Overall, different classifiers led to similar prediction performances, whereas a relatively large variability in performance was observed across different combinations of a resilience dimension and its predicting modality. For example, the physiological features (i.e., ECG, HRV, and GSR) were poor predictors of social competence but strong predictors of social resources during the recall of negative memories. By contrast, speech features (i.e., paralinguistic and linguistic features) were also poor predictors of social competence but strong predictors of structured style during the recall of negative memories. 
Table 3. Results of Resilience Estimation (only negative memories).

\begin{tabular}{|c|c|c|c|c|c|c|c|c|}
\hline \multirow{2}{*}{$\begin{array}{l}\text { Resilience } \\
\text { Dimension }\end{array}$} & \multirow{2}{*}{ Model } & \multicolumn{5}{|c|}{ Single Modality } & \multicolumn{2}{|c|}{ Multiple Modalities } \\
\hline & & GSR & ECG & HRV & Acoustic & LIWC & Physiology & Speech \\
\hline \multirow{4}{*}{ Personal Strength } & KNN & 0.61 & 0.70 & 0.75 & 0.69 & 0.60 & 0.65 & 0.65 \\
\hline & LR & 0.59 & 0.68 & 0.76 & 0.71 & 0.66 & 0.68 & 0.71 \\
\hline & SVC & 0.66 & 0.72 & 0.72 & 0.69 & 0.63 & 0.70 & 0.67 \\
\hline & $\mathrm{RF}$ & 0.66 & 0.72 & 0.72 & 0.67 & 0.63 & 0.70 & 0.64 \\
\hline \multirow{4}{*}{ Family Cohesion } & KNN & 0.63 & 0.64 & 0.70 & 0.75 & 0.69 & 0.68 & 0.74 \\
\hline & LR & 0.42 & 0.68 & 0.69 & 0.71 & 0.68 & 0.57 & 0.69 \\
\hline & SVC & 0.60 & 0.63 & 0.69 & 0.72 & 0.70 & 0.55 & 0.68 \\
\hline & RF & 0.60 & 0.63 & 0.69 & 0.66 & 0.68 & 0.63 & 0.60 \\
\hline \multirow{4}{*}{ Social Resources } & KNN & 0.69 & 0.76 & 0.86 & 0.64 & 0.69 & 0.82 & 0.68 \\
\hline & LR & 0.60 & 0.71 & 0.82 & 0.65 & 0.64 & 0.78 & 0.53 \\
\hline & SVC & 0.73 & 0.73 & 0.84 & 0.67 & 0.65 & 0.79 & 0.63 \\
\hline & $\mathrm{RF}$ & 0.73 & 0.73 & 0.84 & 0.65 & 0.73 & 0.81 & 0.62 \\
\hline \multirow{4}{*}{ Social Competence } & KNN & 0.42 & 0.54 & 0.51 & 0.65 & 0.66 & 0.45 & 0.66 \\
\hline & LR & 0.49 & 0.46 & 0.48 & 0.58 & 0.67 & 0.49 & 0.66 \\
\hline & SVC & 0.46 & 0.50 & 0.49 & 0.60 & 0.70 & 0.47 & 0.63 \\
\hline & RF & 0.49 & 0.46 & 0.48 & 0.62 & 0.68 & 0.50 & 0.63 \\
\hline \multirow{4}{*}{ Structured Style } & KNN & 0.75 & 0.78 & 0.68 & 0.77 & 0.72 & 0.77 & 0.75 \\
\hline & LR & 0.76 & 0.80 & 0.81 & 0.79 & 0.73 & 0.74 & 0.76 \\
\hline & SVC & 0.72 & 0.74 & 0.69 & 0.81 & 0.72 & 0.77 & 0.79 \\
\hline & $\mathrm{RF}$ & 0.72 & 0.74 & 0.69 & 0.79 & 0.76 & 0.79 & 0.78 \\
\hline
\end{tabular}

Note. For each resilience dimension, the best F1-scores for each modality are highlighted in italics, and the best F1-scores across modalities are further highlighted in bold.

Among the single modalities, the HRV and acoustic features are overall the best predictors of resilience across narrative contexts and resilience dimensions. This finding has been hinted at by the relatively large correlations between the HRV/acoustic features and the RSA scores (Figures $4 \mathrm{a}, \mathrm{b}$ and $5 \mathrm{a}, \mathrm{b})$. Note also that predictions that leverage features from multiple modalities were not necessarily better than those using HRV/acoustic features. In the case of physiological signals, adding the poorly performing ECG and GSR features into the augmented feature set might actually decrease the signal-to-noise ratio in the data. In the case of speech signals, the 6373 acoustic features outnumbered the 72 TC-LIWC features in the augmented feature set and might shadow the additional information brought by TC-LIWC features. 
Table 4. Results of Resilience Estimation (positive and negative memories).

\begin{tabular}{|c|c|c|c|c|c|c|c|c|}
\hline \multirow{2}{*}{$\begin{array}{l}\text { Resilience } \\
\text { Dimension }\end{array}$} & \multirow{2}{*}{ Model } & \multicolumn{5}{|c|}{ Single Modality } & \multicolumn{2}{|c|}{ Multiple Modalities } \\
\hline & & GSR & ECG & HRV & Acoustic & LIWC & Physiology & Speech \\
\hline \multirow{4}{*}{ Personal Strength } & KNN & 0.57 & 0.63 & 0.77 & 0.62 & 0.64 & 0.48 & 0.64 \\
\hline & LR & 0.54 & 0.63 & 0.76 & 0.70 & 0.66 & 0.47 & 0.70 \\
\hline & SVC & 0.56 & 0.63 & 0.74 & 0.54 & 0.64 & 0.45 & 0.63 \\
\hline & $\mathrm{RF}$ & 0.56 & 0.63 & 0.74 & 0.64 & 0.66 & 0.44 & 0.67 \\
\hline \multirow{4}{*}{ Family Cohesion } & KNN & 0.56 & 0.63 & 0.65 & 0.56 & 0.58 & 0.50 & 0.56 \\
\hline & LR & 0.43 & 0.68 & 0.68 & 0.68 & 0.55 & 0.43 & 0.66 \\
\hline & SVC & 0.52 & 0.63 & 0.67 & 0.59 & 0.54 & 0.38 & 0.56 \\
\hline & $\mathrm{RF}$ & 0.52 & 0.63 & 0.67 & 0.58 & 0.52 & 0.63 & 0.53 \\
\hline \multirow{4}{*}{ Social Resources } & KNN & 0.60 & 0.72 & 0.85 & 0.64 & 0.63 & 0.51 & 0.66 \\
\hline & LR & 0.53 & 0.66 & 0.80 & 0.67 & 0.65 & 0.47 & 0.68 \\
\hline & SVC & 0.58 & 0.73 & 0.84 & 0.62 & 0.68 & 0.45 & 0.50 \\
\hline & $\mathrm{RF}$ & 0.58 & 0.73 & 0.84 & 0.63 & 0.62 & 0.54 & 0.60 \\
\hline \multirow{4}{*}{ Social Competence } & KNN & 0.37 & 0.43 & 0.57 & 0.69 & 0.58 & 0.41 & 0.71 \\
\hline & LR & 0.48 & 0.46 & 0.53 & 0.69 & 0.57 & 0.30 & 0.71 \\
\hline & SVC & 0.37 & 0.43 & 0.55 & 0.69 & 0.58 & 0.28 & 0.65 \\
\hline & RF & 0.48 & 0.46 & 0.53 & 0.65 & 0.58 & 0.36 & 0.64 \\
\hline \multirow{4}{*}{ Structured Style } & KNN & 0.72 & 0.68 & 0.69 & 0.81 & 0.73 & 0.67 & 0.79 \\
\hline & LR & 0.72 & 0.79 & 0.82 & 0.86 & 0.74 & 0.58 & 0.86 \\
\hline & SVC & 0.74 & 0.69 & 0.69 & 0.81 & 0.72 & 0.69 & 0.75 \\
\hline & RF & 0.74 & 0.69 & 0.69 & 0.78 & 0.73 & 0.71 & 0.76 \\
\hline
\end{tabular}

Note. For each resilience dimension, the best F1-scores for each modality are highlighted in italics, and the best F1-scores across modalities are further highlighted in bold.

\section{Discussion \& Conclusions}

The present study confirms the possibility of estimating personal resilience from speech and physiological signals. Our binary classification of personal resilience successfully achieved F1-scores as high as 0.86 in the cases of predicting social resources by the HRV features during the recall of negative memories and predicting structured style by the paralinguistic features during the recall of positive and negative memories. Our results suggest that the HRV and paralinguistic features are the best predictors of resilience.

For healthcare applications, the HRV data can be conveniently collected from wearable devices, and the paralinguistic features can be collected by a social robot during humanhuman or human-robot conversations to estimate personal resilience. Compared to the extraction of linguistic features, extraction of paralinguistic features does not require automatic speech recognition (ASR), which can be erroneous at times. Therefore, resilience estimation is expected to be more accurate and robust by using the paralinguistic than the linguistic features.

Note that the HRV and paralinguistic features led to comparable prediction performances across positive and negative contexts. It is an important discovery that trait resilience can be well-estimated regardless of narrative contexts. Theoretically, this finding confirms the hypothesis that ostensibly positive events are also relevant in defining resilience [9], which extends the scope of personal resilience. Practically, it is then not required for resilience estimation to actively set up or passively wait for a negative context in which an individual can manifest her/his resilience or lack thereof.

In summary, our research adds to the literature on psychological resilience and personality computing by showing the predictability of personal resilience across different contexts. Automatic assessment of resilience can assist healthcare providers and AI systems/robots in identifying and planning interventions for less resilient individuals. For example, medical practitioners or social robots can be informed to avoid putting too much pressure on individuals low in resilience during interactions, and this vulnerable population can be taught suitable coping strategies for reducing chronic stress. 
To conclude, we hope that this explorative study will arouse the interest of personalitycomputing and social robotics researchers to further advance the automatic recognition of personal resilience. During the COVID-19 pandemic, social distancing prevents people from making social connections, causing loneliness and social isolation. Quarantine may have negative impacts on both physical and mental health and lead to severe consequences, such as a higher rate of suicide. Previous research suggests that resilience can reduce suicidality risk and play a critical role in suicide prevention [57]. Therefore, the predictability of personal resilience can be leveraged to improve one's psychological well-being and has great potential for various mental healthcare applications in the future.

Author Contributions: Conceptualization, S.-M.H. and T.-R.H.; methodology, S.-M.H.; software, S.-H.C.; validation, S.-M.H. and T.-R.H.; formal analysis, S.-M.H.; investigation, S.-M.H.; resources, S.-H.C.; data curation, S.-M.H.; writing-original draft preparation, S.-M.H. and T.-R.H.; writingreview and editing, T.-R.H. and S.-H.C.; visualization, S.-M.H.; supervision, T.-R.H.; project administration, T.-R.H.; funding acquisition, T.-R.H. All authors have read and agreed to the published version of the manuscript.

Funding: This study was financially supported by the grants MOST 110-2634-F-002-040 and MOST 110-2634-F-002-042 from the Ministry of Science and Technology in Taiwan as well as the grants NTU-CC-110L9A00703 and NTU-CC-110L9A00704 from National Taiwan University.

Institutional Review Board Statement: This study was part of a larger human-robot interaction project approved by the Research Ethics Office of National Taiwan University (protocol code: 201803HS017; date of approval: 24 March 2020).

Informed Consent Statement: Informed consent was obtained from all subjects involved in the study.

Data Availability Statement: The data presented in this study are available on request from the corresponding author. The data are not publicly available due to privacy or ethical restrictions.

Acknowledgments: The authors wish to thank Hung-Ping Yeh for helping data collection and Jen-Ho Chang for helpful discussion.

Conflicts of Interest: The authors declare no conflict of interest.

\section{References}

1. Dembling, B.P.; Chen, D.T.; Vachon, L. Life expectancy and causes of death in a population treated for serious mental illness. Psychiatr. Serv. 1999, 50, 1036-1042. [CrossRef]

2. Hiroeh, U.; Appleby, L.; Mortensen, P.B.; Dunn, G. Death by homicide, suicide, and other unnatural causes in people with mental illness: A population-based study. Lancet 2001, 358, 2110-2112. [CrossRef]

3. Hu, T.Q.; Zhang, D.J.; Wang, J.L. A meta-analysis of the trait resilience and mental health. Personal. Individ. Differ. 2015, 76, 18-27. [CrossRef]

4. Heim, C.; Nemeroff, C.B. The role of childhood trauma in the neurobiology of mood and anxiety disorders: Preclinical and clinical studies. Biol. Psychiatry 2001, 49, 1023-1039. [CrossRef]

5. Hovens, J.G.; Wiersma, J.E.; Giltay, E.J.; Van Oppen, P.; Spinhoven, P.; Penninx, B.W.; Zitman, F.G. Childhood life events and childhood trauma in adult patients with depressive, anxiety and comorbid disorders vs. controls. Acta Psychiatr. Scand. 2010, 122, 66-74. [CrossRef]

6. Lauth-Lebens, M.; Lauth, G.W. Risk and resilience factors of post-traumatic stress disorder: A review of current research. Clin. Exp. Psychol. 2016, 2, 1-6. [CrossRef]

7. Masten, A.S. Ordinary magic: Resilience processes in development. Am. Psychol. 2001, 56, 227. [CrossRef]

8. Bonanno, G.A. Loss, trauma, and human resilience: Have we underestimated the human capacity to thrive after extremely aversive events? Am. Psychol. 2004, 59, 20. [CrossRef]

9. Fletcher, D.; Sarkar, M. Psychological resilience. Eur. Psychol. 2013, 18. [CrossRef]

10. Nishimi, K.; Choi, K.W.; Cerutti, J.; Powers, A.; Bradley, B.; Dunn, E.C. Measures of adult psychological resilience following early-life adversity: How congruent are different measures? Psychol. Med. 2020, 1-10. [CrossRef]

11. Davydov, D.M.; Stewart, R.; Ritchie, K.; Chaudieu, I. Resilience and mental health. Clin. Psychol. Rev. 2010, 30, 479-495. [CrossRef] [PubMed]

12. Choi, K.W.; Stein, M.B.; Dunn, E.C.; Koenen, K.C.; Smoller, J.W. Genomics and psychological resilience: A research agenda. Mol. Psychiatry 2019, 24, 1770-1778. [CrossRef] [PubMed]

13. Vinciarelli, A.; Mohammadi, G. A Survey of Personality Computing. IEEE Trans. Affect. Comput. 2014, 5, 273-291. [CrossRef] 
14. Silveira Jacques Junior, J.C.; Gucluturk, Y.; Perez, M.; Guclu, U.; Andujar, C.; Baro, X.; Escalante, H.J.; Guyon, I.; Van Gerven, M.A.J.; Van Lier, R.; et al. First Impressions: A Survey on Vision-based Apparent Personality Trait Analysis. IEEE Trans. Affect. Comput. 2019, 1-20. [CrossRef]

15. Miranda Correa, J.A.; Abadi, M.K.; Sebe, N.; Patras, I. AMIGOS: A Dataset for Affect, Personality and Mood Research on Individuals and Groups. IEEE Trans. Affect. Comput. 2021, 12, 1-15. [CrossRef]

16. Subramanian, R.; Wache, J.; Abadi, M.K.; Vieriu, R.L.; Winkler, S.; Sebe, N. ASCERTAIN: Emotion and Personality Recognition Using Commercial Sensors. IEEE Trans. Affect. Comput. 2018, 9, 147-160. [CrossRef]

17. Scherer, K.R.; Giles, H. Social Markers in Speech; Cambridge University Press: Cambridge, UK, 1979; Volume 6.

18. Mairesse, F.; Walker, M.A.; Mehl, M.R.; Moore, R.K. Using linguistic cues for the automatic recognition of personality in conversation and text. J. Artif. Intell. Res. 2007, 30, 457-500. [CrossRef]

19. Pennebaker, J.W.; Mehl, M.R.; Niederhoffer, K.G. Psychological aspects of natural language use: Our words, our selves. Annu. Rev. Psychol. 2003, 54, 547-577. [CrossRef]

20. Yarkoni, T. Personality in 100,000 Words: A large-scale analysis of personality and word use among bloggers. J. Res. Personal. 2010, 44, 363-373. [CrossRef]

21. Walker, F.R.; Pfingst, K.; Carnevali, L.; Sgoifo, A.; Nalivaiko, E. In the search for integrative biomarker of resilience to psychological stress. Neurosci. Biobehav. Rev. 2017, 74, 310-320. [CrossRef]

22. Li, S.J.; Lu, S.; Ni, S.G.; Peng, K.P. Identifying psychological resilience in Chinese migrant youth through multidisciplinary language pattern decoding. Child. Youth Serv. Rev. 2019, 107, 104506. [CrossRef]

23. Marshall, K.; Abate, A.; Venta, A. Houston Strong: Linguistic markers of resilience after Hurricane Harvey. J. Trauma. Stress Disord. Treat. 2020, 9, e199. [CrossRef]

24. Zohar, A.H.; Cloninger, C.R.; McCraty, R. Personality and heart rate variability: Exploring pathways from personality to cardiac coherence and health. Open J. Soc. Sci. 2013, 1, 32. [CrossRef]

25. Agaibi, C.E.; Wilson, J.P. Trauma, PTSD, and resilience: A review of the literature. Trauma Violence Abus. 2005, 6, 195-216. [CrossRef]

26. Hopper, J.W.; Spinazzola, J.; Simpson, W.B.; van der Kolk, B.A. Preliminary evidence of parasympathetic influence on basal heart rate in posttraumatic stress disorder. J. Psychosom. Res. 2006, 60, 83-90. [CrossRef]

27. Taylor, C.B. Depression, heart rate related variables and cardiovascular disease. Int. J. Psychophysiol. 2010, 78, 80-88. [CrossRef] [PubMed]

28. Moreau, C.; Zisook, S. Rationale for a posttraumatic stress spectrum disorder. Psychiat Clin. N. Am. 2002, 25, 775-790. [CrossRef]

29. Jones, S.E.; LeBaron, C.D. Research on the relationship between verbal and nonverbal communication: Emerging integrations. J. Commun. 2002, 52, 499-521. [CrossRef]

30. Johar, S. Emotion, Affect and Personality in Speech: The Bias of Language and Paralanguage; Springer: Berlin, Germany, 2015.

31. Zhang, T.; Kuo, C.C.J. Content-Based Audio Classification and Retrieval for Audiovisual Data Parsing; Kluwer Academic: Boston, MA, USA, 2013; p. 35.

32. Pennebaker, J.W.; King, L.A. Linguistic styles: Language use as an individual difference. J. Personal. Soc. Psychol. 1999, 77, 1296. [CrossRef]

33. Chung, C.; Pennebaker, J.W. The psychological functions of function words. Soc. Commun. 2007, 1, 343-359.

34. Akinnaso, F.N. On the differences between spoken and written language. Lang. Speech 1982, 25, 97-125. [CrossRef]

35. Smith, B.W.; Dalen, J.; Wiggins, K.; Tooley, E.; Christopher, P.; Bernard, J. The brief resilience scale: Assessing the ability to bounce back. Int J. Behav. Med. 2008, 15, 194-200. [CrossRef] [PubMed]

36. Connor, K.M.; Davidson, J.R. Development of a new resilience scale: The Connor-Davidson Resilience Scale (CD-RISC). Depress. Anxiety 2003, 18, 76-82. [CrossRef]

37. Friborg, O.; Barlaug, D.; Martinussen, M.; Rosenvinge, J.H.; Hjemdal, O. Resilience in relation to personality and intelligence. Int. J. Methods Psychiatr. Res. 2005, 14, 29-42. [CrossRef]

38. Windle, G.; Bennett, K.M.; Noyes, J. A methodological review of resilience measurement scales. Health Qual. Life Outcomes 2011, 9 , 8. [CrossRef]

39. Sutin, A.R.; Robins, R.W. Continuity and correlates of emotions and motives in self-defining memories. J. Personal. 2005, 73, 793-824. [CrossRef]

40. Makowski, D.; Pham, T.; Lau, Z.J.; Brammer, J.C.; Lespinasse, F.; Schoelzel, C.; Chen, S.A. NeuroKit2: A python toolbox for neurophysiological signal processing. Behav. Res. Methods 2021, 53, 1689-1696. [CrossRef]

41. Gabrieli, G.; Azhari, A.; Esposito, G. PySiology: A python package for physiological feature extraction. In Neural Approaches to Dynamics of Signal Exchanges; Springer: Berlin, Germany, 2020; pp. 395-402.

42. Schuller, B.; Steidl, S.; Batliner, A.; Vinciarelli, A.; Scherer, K.; Ringeval, F.; Chetouani, M.; Weninger, F.; Eyben, F.; Marchi, E. The INTERSPEECH 2013 computational paralinguistics challenge: Social signals, conflict, emotion, autism. In Proceedings of the 14th Annual Conference of the International Speech Communication Association, Lyon, France, 25-29 August 2013.

43. Eyben, F.; Wöllmer, M.; Schuller, B. Opensmile: The munich versatile and fast open-source audio feature extractor. In Proceedings of the 18th ACM International Conference on Multimedia, Firenze, Italy, 25-29 October 2010; pp. 1459-1462. 
44. Eyben, F.; Scherer, K.R.; Schuller, B.W.; Sundberg, J.; André, E.; Busso, C.; Devillers, L.Y.; Epps, J.; Laukka, P.; Narayanan, S.S. The Geneva minimalistic acoustic parameter set (GeMAPS) for voice research and affective computing. IEEE Trans. Affect. Comput. 2015, 7, 190-202. [CrossRef]

45. Li, P.-H.; Fu, T.-J.; Ma, W.-Y. Why Attention? Analyze BiLSTM Deficiency and Its Remedies in the Case of NER. In Proceedings of the Thirty-Fourth AAAI Conference on Artificial Intelligence, New York, NY, USA, 7-12 February 2020; pp. 8236-8244.

46. Kutner, M.H.; Nachtsheim, C.J.; Neter, J.; Li, W. Applied Linear Statistical Models; McGraw-Hill: New York, NY, USA, 2005.

47. Oshio, A.; Taku, K.; Hirano, M.; Saeed, G. Resilience and Big Five personality traits: A meta-analysis. Personal. Individ. Differ. 2018, 127, 54-60. [CrossRef]

48. Chida, Y.; Hamer, M. Chronic psychosocial factors and acute physiological responses to laboratory-induced stress in healthy populations: A quantitative review of 30 years of investigations. Psychol. Bull. 2008, 134, 829. [CrossRef]

49. Lampert, R. ECG signatures of psychological stress. J. Electrocardiol. 2015, 48, 1000-1005. [CrossRef] [PubMed]

50. Norris, C.J.; Larsen, J.T.; Cacioppo, J.T. Neuroticism is associated with larger and more prolonged electrodermal responses to emotionally evocative pictures. Psychophysiology 2007, 44, 823-826. [CrossRef]

51. Harnmerschmidt, K.; Juergens, U. Acoustical correlates of affective prosody. J. Voice 2007, 21, 531-540. [CrossRef] [PubMed]

52. Fredrickson, B.L.; Tugade, M.M.; Waugh, C.E.; Larkin, G.R. What good are positive emotions in crises? A prospective study of resilience and emotions following the terrorist attacks on the United States on September 11th, 2001. J. Personal. Soc. Psychol. 2003, 84, 365-376. [CrossRef]

53. Pennebaker, J.W.; Boyd, R.L.; Jordan, K.; Blackburn, K. The Development and Psychometric Properties of LIWC2015; The University of Texas at Austin: Austin, TX, USA, 2015.

54. Tausczik, Y.R.; Pennebaker, J.W. The psychological meaning of words: LIWC and computerized text analysis methods. J. Lang. Soc. Psychol. 2010, 29, 24-54. [CrossRef]

55. Tugade, M.M.; Fredrickson, B.L. Resilient individuals use positive emotions to bounce back from negative emotional experiences. J. Personal. Soc. Psychol. 2004, 86, 320-333. [CrossRef] [PubMed]

56. Waugh, C.E.; Thompson, R.J.; Gotlib, I.H. Flexible Emotional Responsiveness in Trait Resilience. Emotion 2011, 11, 1059-1067. [CrossRef] [PubMed]

57. Johnson, J.; Wood, A.M.; Gooding, P.; Taylor, P.J.; Tarrier, N. Resilience to suicidality: The buffering hypothesis. Clin. Psychol. Rev. 2011, 31, 563-591. [CrossRef] 